\title{
High Sensitive Mass Detection using GaAs Coupled Micro Resonators
}

\section{Tony Chopard ${ }^{1}$ and Therese Leblois ${ }^{1}$ *}

1 FEMTO-ST Institute, University of Franche-Comté, 32 avenue de l'observatoire, 25044 Besançon cedex, France; E-Mails: therese.leblois@ femto-st.fr, tony.chopard@femto-st.fr

* Therese Leblois; therese.leblois@ @emto-st.fr;

Tel.: +33-381-853-974; Fax: +33-381-853-998.

Published: 1 June 2014

\begin{abstract}
This work demonstrates the improvement of mass detection sensitivity and time response using a quite simple structure of sensor. Indeed, complicated technological processes are often required to reach high sensitivity when we want to detect specific molecules in biological fields. These developments constitute an obstacle to the early diagnosis of diseases. An alternative is the design of coupled structures. The device is based on the piezoelectric excitation and detection of two GaAs micro structures vibrating on antisymmetric modes. GaAs is a material which has the advantage to be micromachined easily using clean room processes. Moreover, we showed its high potential in direct biofunctionalisation to be used in biological field. A specific design of the device was performed to improve the detection at low mass and an original detection method has been developed. The principle is to exploit the variation in amplitude at the initial resonance frequency which has in the vicinity of weak added mass the greatest slope. Therefore, we get a very good resolution for an infinitely weak mass: relative voltage variation of $8 \% / 1 \mathrm{fg}$. The analysis is based on results obtained by finite element simulation.
\end{abstract}

Keywords: GaAs piezoelectric transducer; acoustic coupling; high sensitivity;

\section{Introduction}

Low mass detectors are becoming highly desirable, mainly in the biology and environment fields to detect small objects, like molecules or atoms [1-3]. For example, the field of biology requires mass detectors with a high resolution, especially in the range [1zg, 10fg], to detect the presence of particles 
(grafting onto a surface, deposition, adsorption). However, measurement mass techniques by conventional sensors require an important miniaturization to get sensitivity in the zeptogram range [16]. These manufacturing techniques are complicated to develop and control. Moreover, the sensing structures will reach a nanometer size and then will become very brittle. That's why coupled sensor networks arouse, since recently, a particular enthusiasm [7-15]. Coupled structures present also other advantages such as the opportunity to perform on the same substrate differential measurements or to obtain a multiplexed analysis of a biological or chemical solution. The choice of GaAs as the material for the transducer is due to first a good knowledge of its microfabrication technology and second to the opportunity to biofunctionalize this material. In parallel, we proved the biocompatibility of GaAs surface for in vitro analysis [16-18]. The work presented here constitutes one of the preliminary steps necessary before the integration of electronics and transducers arrays on the same substrate for biological field measurements. The paper is divided into three sections. First, we present the principle of the coupled resonators and the usual method of mass measurement. In the second section, we compare different methods of analysis to improve the sensitivity and time response. Finally, we propose the design of our device.

\section{Measurement principle and results}

\subsection{Principle}

We consider a tuning fork in GaAs crystal with coupled resonant cantilevers vibrating on an antisymmetric bending mode. The size of the device is given on figure 1. With this geometry, the resonance frequency $f_{R}$ is obtained at $f_{R}=109 \mathrm{kHz}$.

Figure 1. Design of the coupled microcantilevers. $L=25 \mu \mathrm{m}, \mathrm{w}=10 \mu \mathrm{m}, \mathrm{th}=0.1 \mu \mathrm{m}, \mathrm{p}=20 \mu \mathrm{m}$ and $\mathrm{b}=8 \mu \mathrm{m}$

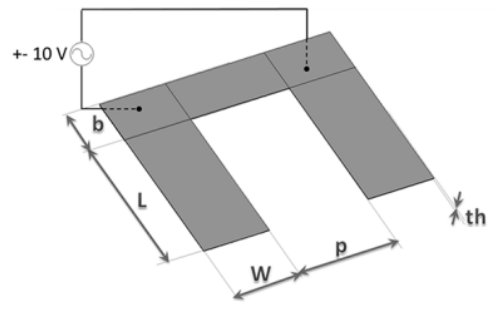

The frequency response is performed to identify, without and with added mass, the maximum amplitude of the resonance peak. The usual method consists in the experimental determination of the frequency shift $\Delta f$ of the resonance frequency $f_{R}$ with the added mass $\Delta m$. The expression of $\Delta f$ is given in equation (1). It depends on the resonator mass $m$ and we can observe that the increase of sensitivity passes through a decrease of mass. So, the variation of amplitude $\Delta u$ is an image of the added mass $\Delta m$ in a quasi-linear function as seen in equation (2). This last method is preferred to the frequency shift determination if $\mathrm{k}_{\mathrm{c}}<\mathrm{k} / 2$.

$$
\frac{\Delta f}{f_{R}} \approx \frac{\Delta m}{2 \times m}
$$




$$
\frac{\Delta u}{u_{R}} \approx \frac{\Delta m \times k}{4 \times k_{c} \times m} \quad,\left\{\begin{array}{l}
k_{c}: \text { coupling spring } \\
k: \text { cantilever spring }
\end{array}\right.
$$

We performed calculation on an antisymmetric bending mode for which the amplitude variation is higher than that of the symmetric mode. As well as in the publication of E. Gil-Santos et al [12], we observed a quasi-linear decrease of the amplitude as a function of the added mass. We obtained in the range $[0,150 \mathrm{fg}]$, a linear relative variation in resonance peak amplitude of $8000 \mathrm{ppm} / \mathrm{fg}$ against 2 $\mathrm{ppm} / \mathrm{fg}$ for the relative variation in resonance frequency. These values confirm the advantages of the amplitude method for coupled microcantilevers.

Nethertheless the sensitivity is not sufficient to detect biological molecules. We propose to improve the performance using different methods to exploit the frequency versus mass spectrum.

\subsection{Methods of analysis for resonant amplitude versus frequency curves}

In the previous section, we propose two methods:

- A-Method: determination of the frequency shift with added mass.

- B-Method: determination of the amplitude at the resonance peak by frequency sweeping.

Fig. 2(a) shows the evolution of $\Delta f$ and $\Delta u$ with $\Delta m$. As already say, the relative variation of amplitude is a better method in the case of coupled cantilevers. As seen on Fig.2(b) we have an overlap of resonance peak for a very weak added mass and so, it remains difficult to reach the required sensitivity. To improve performances using the same measurements principle as previously, we propose, for very weak mass to determine the variation of the amplitude at the fixed frequency $f_{R}$ where $f_{R}$ is the resonant frequency at the initial weight $m$. This method is called C-method. Compared to $\mathrm{A}$ and B-methods, a significant increase of the relative variation in the amplitude is obtained (Fig.2(c)). Moreover, as the frequency sweep can be omitted, the measurement process is easier and the acquisition time shorter.

It is clear that the higher the quality factor, the higher the sensitivity of the transducer. The operating range which is one of the criteria for transducer characterization is given by equation (3). It can be deduced from the initial microcantilever mass $m$, the resonance frequency of the selected mode $f_{R}$ and the half-width of the resonance peak at $10 \%$ of the relative variation in amplitude.

$$
\text { Range } \approx \frac{m \times \Delta f_{(\Delta \text { relative amplitude change }=10 \%)}}{f_{R}}
$$

The C-method is valid as long as there is a monotonic overlap of the resonance peak with added mass on the resonance characteristic at initial weight. In the case of Fig.2(d), we have $\Delta f$ (Arelative amplitude change $=10 \%)=4 \mathrm{~Hz}$ and the operating range is equal to $15 \mathrm{fg}$. It may be noticed that if the resonance curve has a better quality factor, then the resolution increases but the operating range decreases. As the operating range is too tiny, we propose to use a hybrid method to exploit the good performances of C-method when we are concerned with very weak added mass. It consists without modifying the device to use Bmethod when the added mass is over the operating range. With our device, the threshold for switching between the two methods is for $\Delta m=15 \mathrm{fg}$ which corresponds to a relative variation in the maximum deflection of $11.5 \%$. 
Figure 2. (a) B-method / A-Method vs added mass: comparison between the relative variation of the maximum amplitude of the beam deflection and the relative variation of the frequency shift of the resonance peak. (b) Amplitude without and with added mass vs frequency. (c) Zoom on the overlap shown in (b). We exploit the resonance peak overlap to obtain a higher resolution with C-method than with A and B methods. (d) Amplitude vs frequency around resonance frequency: determination of the sensor range.

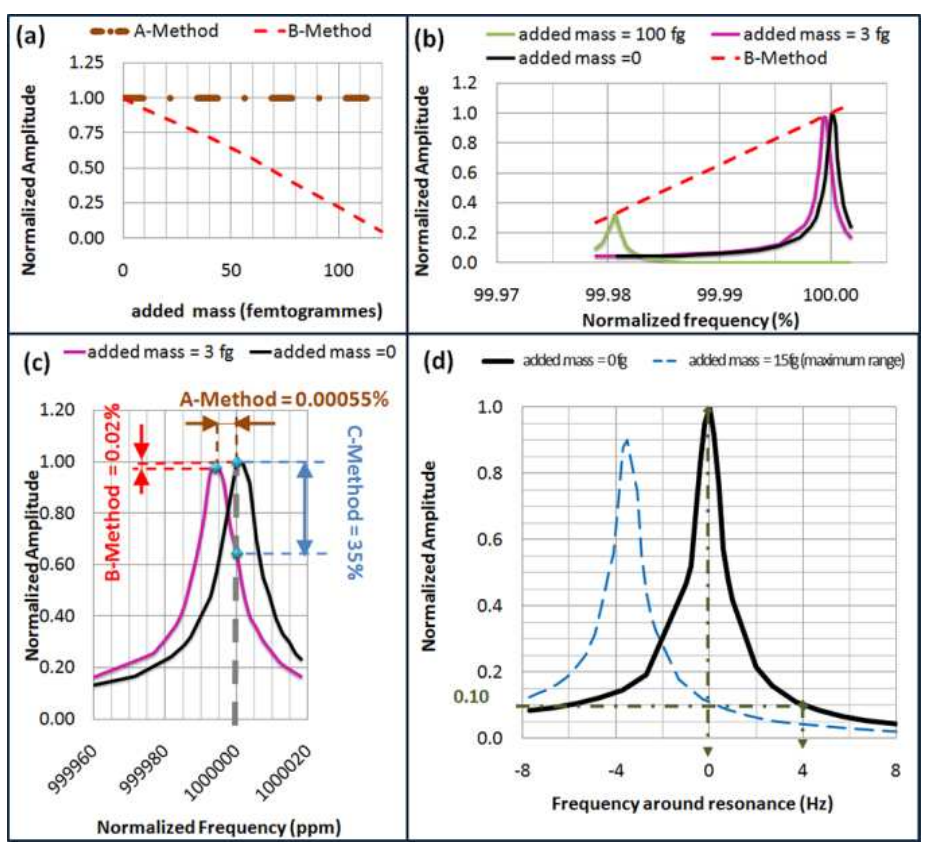

\subsection{Design of the device}

To adapt the previous methods we used a device with a piezoelectric excitation and detection. The main advantage of the piezoelectric measurement with respect to the optical one is to be able to obtain a resonance peak with the appearance of an even or odd differentiable function according to the electrode position on the coupling element. According to a study parameterized in frequency on the voltage change within the coupling element, we determined that we need to deposit an electrode at the coupling element center of the microcantilevers. As shown in Fig.3(a), this electrode gives a response with an even function. Nevertheless, an off center electrode provides an odd function. As the tangent at zero of an odd differentiable function is significantly greater than an even differentiable function, it's undeniably preferable to exploit the response of the off center electrode for an infinitely weak mass following the C-method. The device (Fig.3(b)) is then composed of three electrodes, the balancing electrode keeping the symmetry of the device.

Figure 3. (a) Frequency analysis of voltage signals Vce and Voce. (b) Piezoelectric excitation and detection with three electrodes placed at regular intervals and centered on the coupling element.

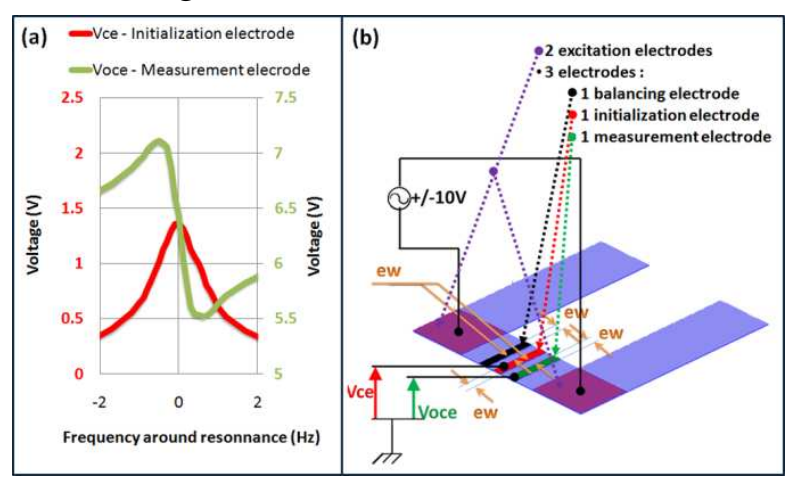


With this device, we obtained in the range from $1 \mathrm{zg}$ to $0.1 \mathrm{fg}$ a resolution 36 times higher than with the B-method. This corresponds to a relative variation in voltage of $8 \% / f g$ with is a very good sensitivity for the infinitely weak mass according to the size of the device. To expand the operating range we used as already seen the hybrid method of analysis.

\section{Conclusions/Outlook}

To conclude, we have shown that the sensitivity of mass sensors based on coupled cantilevers can be significantly enhanced by a thorough analysis of the measurement. We have proposed and demonstrated the benefit of an analysis method which significantly increases the resolution for the addition of very weak mass on coupled microcantilevers. Moreover, utilizing such a method for mass detection offers a real advantage over the more conventional resonant frequency shift approach and the maximum amplitude method. Indeed, thanks to this attractive method of analysis, the fabrication of highly miniaturized devices can be avoided. Optical and piezoelectric detections have been considered and compared in terms of sensitivity at very weak added mass. The proposed method is particularly efficient for piezoelectric detection using appropriate electrodes design. The results with this method are very promising and the device remains easily to microfabricate. In the future, we like to achieve coupled sensors whose structure is based on membranes instead of beams to change the mode of vibration, increase the resonant frequency and improve the quality factor.

\section{Conflicts of Interest}

The authors declare no conflict of interest.

\section{References}

1. Battiston, F. M.; Ramseyer, J. P.; Lang, H. P.; Baller, M. K.; Gerber Ch.; Gimzewski, J. K.; Guntherodt, H. J.; A chemical sensor based on a microfabricated cantiler array with simultaneous resonance-frequency and bending readout, Sensors and Actuators B 2001, 77, 122-131.

2. Rogers, B.; Manning, L.; Jones, M.; Sulcheck, T.; Murray, K.; Beneschott, B.; Adams, J. D.; Hu, Z.; Thundat, T.; Cavazos, H.; Minne, S. C.; Mercury vapor deposition with a self-sensing resonating piezoelectric cantilever, Review of Scientific Instruments 2003, 74, 4899-4902.

3. Mertens, J.; Finot, E.; Nadal, M. H.; Eyraud, V.; Heintz, O.; Bourillot, E.; Detection of gas trace of hydrofluoric acid using microcantilever, Sensors and Actuators B 2004, 99, 58-65.

4. Yang, Y.; Callegari, C.; Feng, X.; Ekinci, K.; Roukes, M.; Zeptogram-scale nanomechanical mass sensing, Nano letters 2006, 6, 583-586.

5. Jensen, K.; Kim, K.; Zettl, A.; An atomic resolution nanomechnical mass sensor, Nature nanotechnology 2008, 3, 533-537.

6. Gil-Santos, E.; Ramos, D.; Martinez, J.; Fernandez-Regulez, M.; Garcia, R.; San Paulo, A.; Calleja, M.; Tamayo, J.; Nanomechnanical mass sensing and stiffness spectrometry based on twodimensional vibrations of resonant nanowires, Nature Nanotechnology 2010, 5, 641-645. 
7. Spletzer, M.; Raman, A.; Sumali, H.; Sullivan, J.; Highly sensitive mass detection and identification using vibration localization in coupled microcantilever arrays, Applied Physics Letters 2008, 92(11), 114102-105.

8. DeMartini, B. E.; Rhoads, J. F.; Shaw, S. W.; Turner, K. L.; A single inputs-single output mass sensor based on a coupled array of microresonators, Sensors and actuators A 2007, 137, 147-156.

9. Thiruvenkatanathan, P.; Yan, J.; Woodhouse, J.; Seshia, A.; Enhancing parametric sensitivity in electrically coupled MEMS resonators, Journal of Microelectromechanical System 2009, 18(5), 1077-1086.

10. Karabalin, R.; Cross, M.; Roukes, M.; Nonlinear dynamics and chaos in two coupled nanomechanical resonators, Physical Review B 2009, 79(16), 165309-14.

11. Okamoto, H.; Kitajima, N.; Onomitsu, K.; Kometani, R.; Warisawa, S.; Ishihara, S.; Yamaguchi, H.; High sensitivity charge detection using antisymmetric vibration in coupled micromechanical oscillators, Applied Physics Letters 2011, 98(1), 014103-06.

12. Gil-Santos, E.; Ramos, D.; Pini, V.; Calleja, M.; Tamayo, J.; Exponential tuning of the coupling constant of coupled microcantilevers by modifying their separation, Applied Physics Letters 2011, 98(12), 123108-111.

13. Huber, T. M.; Abell, B. C.; Mellema, D. C.; Spletzer, M.; Raman, A.; Mode-selective non-contact excitation of microcantilevers and microcantilever arrays in air using the ultrasound radiation force, Applied Physics Letters 2010, 97(21), 214101-104.

14. Chopard, T.; Bienaime, A. Elie-Caille, C.; Leblois, T.; High sensitive mass detection using piezoelectric coupled microcantilevers, Proceedings of Eurosensors XXVI, Krakow, Poland, September 9-12 2012, Elsevier, Procedia Engineering 2012, 47, 350-353.

15. Thiruvenkatanathan, P.; Yan, J.; Woodhouse, J.; Aziz, A.; Seshia, A.; Ultrasensitive modelocalized mass sensor with electrically tunable parametric sensitivity, Applied Physics Letters 2010, 96(8), 081913-916.

16. Bienaime, A .; Microcapteur GaAs pour la détection de molécules dans un fluide biologique, $\mathrm{PhD}$ level, University of Franche-Comté, France, December 112011.

17. Dubowski, J. J.; Voznyy, O.; Marshall, G. M.; Molecular Self-assembly and Passivation of GaAs(001) with Alkanethiol Monolayers: A View Towards Bio-functionalization, Applied Surface Science 2010, 256, 5714-5721.

18. Bienaime, A.; Leblois, T.; Gremaud, N.; Chaudon, M. J. ; El Osta, M. ; Pecqueur, D. ; Ducoroy, P. ; Elie-Caille, C. ; Influence of a thiolate chemical layer on $\operatorname{GaAs}(100)$ biofunctionalization : an original approach coupling AFM and mass spectrometry methods, Materials 2013, 6(11), 49464966.

(C) 2014 by the authors; licensee MDPI, Basel, Switzerland. This article is an open access article distributed under the terms and conditions of the Creative Commons Attribution license (http://creativecommons.org/licenses/by/3.0/). 